\title{
Estimating Arterial Wall Impedance using a Plethysmogram
}

\author{
Akira Sakane, Toshio Tsuji, and Yoshiyuki Tanaka \\ Department of the Artificial Complex Systems Engineering \\ Hiroshima University \\ Higashi-Hiroshima, 739-8527 JAPAN \\ sakane@bsys.hiroshima-u.ac.jp \\ tsuji@bsys.hiroshima-u.ac.jp \\ ytanaka@bsys.hiroshima-u.ac.jp
}

\author{
Noboru Saeki and Masashi Kawamoto \\ Graduate School of Biomedical Sciences \\ Hiroshima University \\ Kasumi 1-2-3, Minami-ku, Hiroshima, 734-8551 JAPAN \\ nsaeki@hiroshima-u.ac.jp \\ anekawa@hiroshima-u.ac.jp
}

\begin{abstract}
This paper proposes a new method to estimate the mechanical impedance of arterial walls with biological signals, such as electrocardiogram, arterial pressure, and pulse oximetric plethysmogram that reflects arterial pulsation. A mathematical model of the fingertip arterial wall is developed on the basis of the relationship between the arterial pressure and the plethysmogram. The validity of the proposed method is then discussed by applying to monitor the arterial wall impedance properties during surgical operations. It has been demonstrated that the changes of the measured impedance parameters correspond to events during operations.
\end{abstract}

\section{INTRODUCTION}

The vascular system spreads through the body and transports various substances necessary for life, such as oxygen, nutrition, and so on. Pulsation in company with blood flow is effectively absorbed and moderated by the highly elastic aorta, so that blood flows smoothly even in capillaries [1]. However, when arterial compliance degrades due to aging, the aorta cannot regulate pulsation efficiently. Because of aging and the changes of lifestyle in recent years, cardiac and cerebrovascular diseases caused by the degradation of vascular performance have tended to increase.

Vascular properties are affected and changed by a number of factors, which are roughly divided into organic and functional changes. Organic change is mainly caused by aging, and its degeneration leads to many diseases. For instance, cerebral infarction often results from becoming narrow arterial lumen because of cerebral atherosclerosis. In contrast, functional change is to be stiff the blood vessel by losing elasticity, in which pulsation is not effectively absorbed. Quantitative evaluation for these changes of vascular properties may be useful for preventing disease and monitoring health.

This paper introduces mechanical impedance as a quantitative index of dynamic characteristics of the blood vessel. Mechanical impedance, consisting of stiffness, viscosity, and inertia, has been extensively used in models of biological dynamic characteristics. For example, Mussa lvaldi et al. pioneered the measurement of human hand stiffness, and examined the hand stiffness in a stable arm posture [2]. Also, Dolan et al. estimated not only hand stiffness but also viscosity and inertia [3], and Tsuji et al. measured impedances under various conditions on the muscle contraction level, arm posture and motion direction [4], [5]. Oka modeled the hardness of the skin surface using mechanical impedance [6]. Since the arterial wall includes smooth muscle, it is also possible to express dynamic characteristics of arterial walls by mechanical impedance.

Many techniques have been applied to measure arterial wall stiffness. For example of invasive methods to measure the stiffness in the 1960's, the arterial wall stiffness has been measured during open-heart surgery in which the aorta is exposed [7]. In this approach, variations in blood pressure and vessel diameter were measured by hemodynamometer and calipers, respectively, to estimate the stiffness. However, it is almost impossible to use such an invasive approach on healthy individuals because of ethical problems. Also, some researches have attempted to describe vascular dynamic characteristics by a non-invasive approach. For example, Katayama et al. estimated the compliance of the arterial wall using a plethysmogram and blood pressure [8], and other similar experiments that estimated the arterial wall compliance have also been reported [9], [10]. These researches, however, dealt with only stiffness, and did not provide sufficient analysis of vascular characteristics. Mascaro and Asada et al. reported the estimated values of mechanical impedance of the arterial wall from the velocity of blood flow and arterial caliber, but did not discuss the accuracy [11]. Kato et al. provided a vascular model based on the Voigt model, which is suited for describing vascular smooth muscle mechanics, and the vascular conditions were estimated using stiffness and viscosity [12]. However, this model does not consider inertia properties, and the accuracy is not so good. Moreover, Murahara et al. presented a cardiovascular system using a model based on hemodynamics, and estimated the changes of pressure waveforms in simulation [13]. Their model, however, had not been calibrated with real data.

As the first step to estimating vascular mechanical properties non-invasively, the present paper proposes a method to express the dynamic characteristics of the arterial wall using mechanical impedance (i.e., stiffness, viscosity, and inertia) and estimates the vascular conditions beat-to-beat. In the proposed method, the impedance parameters can be estimated from an electrocardiogram, blood pressure, and plethysmogram by the linear least-squares method. 


\section{IMPEDANCE MODEL OF ARTERIAL WALL}

\section{A. Arterial Wall Model}

The anatomical structure of the arterial wall is depicted in Fig. 1 [14]. The artery has high elasticity and is controlled by the sympathetic nervous system and hormones such as angiotensin II through intervention stimulations [1].

The arterial wall is constructed in three layers: the tunica adventitia, the tunica media, and the tunica intima. The tunica adventitia contains a mechanical factor that affect artery stiffness and compliance such as connective tissue, elastin, and collagen. The tunica media consists of elastin, collagen, and much smooth muscle, that is contracted by stimulations of sympathetic nerves and hormones such as norepinephrine and angiotensin II, and it makes blood flow down. The tunica intima consists of the vascular endothelia that produce endothelium-derived vasoactive substances, such as NO (nitric oxide) and EDHF (endothelium-derived hyperpolarizing factor) [15]. Therefore, vascular contraction or relaxation is controlled by smooth muscle in the tunica media. The present paper models dynamic characteristics of the arterial wall with mechanical impedance. The dynamic characteristics of the arterial wall are analyzed by applying the impedance estimation method of skeletal muscle considered stiffness, viscosity, and inertia.

Fig. 2 illustrates the proposed impedance model of the arterial wall. This model considers only the characteristics of the arterial wall in the arbitrary radius direction. The impedance characteristic can be described using external force and displacement of arterial wall as follows:

$$
F(t)=M \ddot{r}(t)+B \dot{r}(t)+K\left(r(t)-r_{e}\right)
$$

where $F(t)$ is force applying the arterial wall by blood flow; $M, B$, and $K$ are inertia, viscosity, and stiffness, respectively; $r(t), \dot{r}(t)$, and $\ddot{r}(t)$ are position, velocity, and acceleration of the wall, respectively; and $r_{e}$ denotes the equilibrium vascular radius when blood pressure is zero. The vascular dynamic characteristic at the time $t$ can be derived as follows:

$$
d F(t)=M d \ddot{r}(t)+B d \dot{r}(t)+K d r(t)
$$

where $d r(t)=r(t)-r\left(t_{0}\right) ; d \dot{r}(t)=\dot{r}(t)-\dot{r}\left(t_{0}\right) ; d \ddot{r}(t)=$ $\ddot{r}(t)-\ddot{r}\left(t_{0}\right) ; d F(t)=F(t)-F\left(t_{0}\right)$; and $t_{0}$ denotes the start time just at moving arterial walls.

To estimate the impedance parameters based on (2), it is necessary to measure $F(t)$ and $r(t)$. Assuming that blood pressure and force are proportional for simplification, the following equation can be obtained:

$$
F(t)=k_{f} P_{b}(t)
$$

where $k_{f}$ is a proportional constant, and $P_{b}(t)$ is blood pressure.

On the other hand, the vascular radius $r(t)$ is quite difficult to be measured directly, so a plethysmogram is utilized instead of $r(t)$.

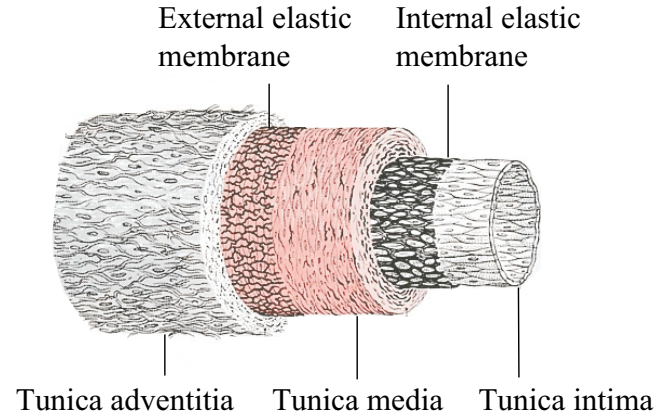

Fig. 1. Arterial wall ${ }^{[14]}$

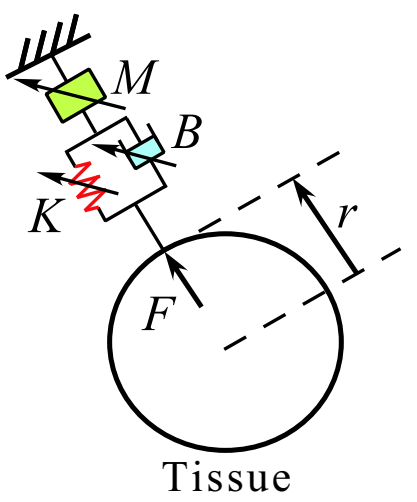

Fig. 2. Arterial wall impedance model

\section{B. Plethysmogram}

The variation of blood flow by pulsation of the heart is transmitted peripherally in the arteries and can be measured as a pulse wave. There are two types of the pulse waves: sphygmogram representing variations of pressure, and plethysmogram representing variations of volume.

Let $I_{0}$ denote the intensity of incident light on a blood vessel with diameter $D$, and $I_{D}$ denote the intensity of light transmitted through the apex of the finger. According to Lambert-Beer's law [16], the following equation can be obtained [17]:

$$
A_{D} \equiv \log \left(I_{0} / I_{D}\right)=E C D
$$

where $A_{D}$ is the optical density that is proportional to the concentration of absorptive substance $C$ and the diameter of a blood vessel $D . E$ is the peculiar absorptive constant for each material. If the case that the caliber of a blood vessel $D$ changes $D+\Delta D(t)$ and the light transmitted through the apex of the finger becomes $I_{D}-\Delta I(t)$, the variation of optical density $\Delta A(t)$ can be expressed as

$$
\begin{aligned}
\Delta A(t) & =A(t)-A_{D} \\
& =\log \left(I_{D} /\left(I_{D}-\Delta I(t)\right)\right)=E C \Delta D(t) .
\end{aligned}
$$

The variation of optical density $\Delta A(t)$ is defined as the 
plethysmogram $P_{l}(t)$. In (5), $\Delta A(t)$ cannot be calculated directly since the equation has an indefinite component $C$ that relies on blood concentration.

The method for estimating the variation of optical density is explained in Fig. 3 [17]. In the figure, $I_{s}$ and $I_{d}$ denote the intensity of transmitted light through the apex of the finger in systolic and diastolic phases, respectively. The variation of optical density in systolic and diastolic phases, $\Delta A_{s}(t)$ and $\Delta A_{d}(t)$, can be given as follows [17]:

$$
\begin{aligned}
\Delta A_{s}(t)= & -\log \left(1-\Delta I_{s}(t) / I_{D}\right) \\
= & \Delta I_{s}(t) / I_{D}+\left(\Delta I_{s}(t) / I_{D}\right)^{2} / 2+\cdots \\
& +\left(\Delta I_{s}(t) / I_{D}\right)^{n} / n+\cdots \\
\Delta A_{d}(t)= & -\log \left(1+\Delta I_{d}(t) / I_{D}\right) \\
= & -\Delta I_{d}(t) / I_{D}+\left(\Delta I_{d}(t) / I_{D}\right)^{2} / 2+\cdots \\
& -(-1)^{n-1}\left(\Delta I_{d}(t) / I_{D}\right)^{n} / n+\cdots
\end{aligned}
$$

where $\Delta I_{s}(t)=I_{D}-I_{s}(t)$ and $\Delta I_{d}(t)=I_{d}(t)-I_{D}$. It should be noted that $\Delta I_{s}(t)=0$ in the diastolic phase and $\Delta I_{d}(t)=0$ in the systolic phase. The variation of optical density $\Delta A(t)$ can then be represented as follows [17]:

$$
\begin{aligned}
\Delta A(t)= & \Delta A_{s}(t)-\Delta A_{d}(t) \\
= & \Delta I(t) / I_{D}+\left(\Delta I^{\prime}(t) / I_{D}\right)^{2} / 2+\cdots \\
& +\left(\Delta I^{\prime}(t) / I_{D}\right)^{2 n} / 2 n+\cdots
\end{aligned}
$$

where $\Delta I(t)=\Delta I_{s}(t)+\Delta I_{d}(t) ; \Delta I^{\prime}(t)=\Delta I_{s}(t)-\Delta I_{d}(t)$. Since $\Delta I(t) / I_{D}$ and $\Delta I^{\prime}(t) / I_{D}$ are small enough, the terms higher than the second order can be omitted [17]. As a result, the plethysmogram is expressed by

$$
\begin{aligned}
P_{l}(t) \equiv \Delta A(t) & =\log \left(I_{D} /\left(I_{D}-\Delta I(t)\right)\right) \\
& \simeq \Delta I(t) / I_{D} .
\end{aligned}
$$

In this paper, it is assumed that $A(t)$ is proportional to the summation of the vascular radius in the measured part:

$$
A(t)=k_{p} r_{v}(t)
$$

where $k_{p}$ is the proportional constant and $r_{v}(t)$ is the virtual vascular radius. Thus, from (5), (9), and (10), the virtual vascular radius can be derived as follows:

$$
r_{v}(t)=\frac{P_{l}(t)+A_{D}}{k_{p}} .
$$

\section{Impedance Model}

The force exerted on the arterial wall is expressed by the arterial pressure $P_{b}(t)$ given by (3), and the virtual vascular radius is represented by the plethysmogram $P_{l}(t)$ in (11). The arterial wall impedance is then estimated using $P_{b}(t)$ and $P_{l}(t)$.

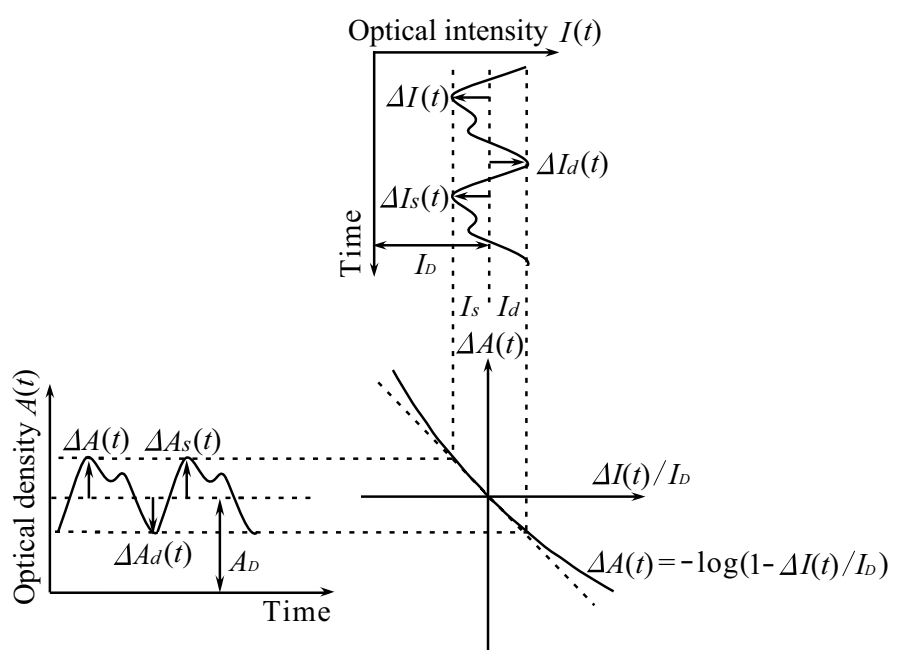

Fig. 3. Conception of plethysmogram [17]

Substituting $r_{v}(t)$ in (11) into $r(t)$ in (2), the vascular dynamic characteristic can be derived from (2) as follows:

$$
d P_{b}(t)=\tilde{M} d \ddot{P}_{l}(t)+\tilde{B} d \dot{P}_{l}(t)+\tilde{K} d P_{l}(t)
$$

where $d P_{b}(t)=P_{b}(t)-P_{b}\left(t_{0}\right), d P_{l}(t)=P_{l}(t)-P_{l}\left(t_{0}\right)$, $d \dot{P}_{l}(t)=\dot{P}_{l}(t)-\dot{P}_{l}\left(t_{0}\right)$, and $d \ddot{P}_{l}(t)=\ddot{P}_{l}(t)-\ddot{P}_{l}\left(t_{0}\right)$. Also, the impedance parameters included in (12) are given as

$$
\begin{aligned}
\tilde{M} & =\frac{M}{k_{p} k_{f}} \\
\tilde{B} & =\frac{B}{k_{p} k_{f}} \\
\tilde{K} & =\frac{K}{k_{p} k_{f}} .
\end{aligned}
$$

Consequently, the impedance parameters $\tilde{M}, \tilde{B}$, and $\tilde{K}$ can be estimated by measuring $P_{b}(t)$ and $P_{l}(t)$.

\section{IMPEDANCE ESTIMATION EXPERIMENTS}

\section{A. Experimental Method}

Figure 4 describes the apparatus used in the experiment. The arterial pressure was measured through a catheter ( 24 gauge) placed in the left radial artery, and the plethysmogram was measured with the ipsilateral forefinger (BSS-9800, NIHON KOHDEN Co., Ltd).

The impedance parameters are estimated in the following steps: Electrocardiogram $(E C G(t))$, arterial pressure $\left(P_{b}(t)\right)$, and plethysmogram $\left(P_{l}(t)\right)$ were sampled at $125[\mathrm{~Hz}]$, and were recorded simultaneously in a personal computer for offline analysis. The data were preprocessed and used to estimate the impedance parameters with the least squares method. In order to ascertain the estimation accuracy, the proposed method was applied to endoscopic transthoracic sympathectomy to estimate impedance parameters, and hyperhidrosis patient's data were examined (Patient A). The data were measured 

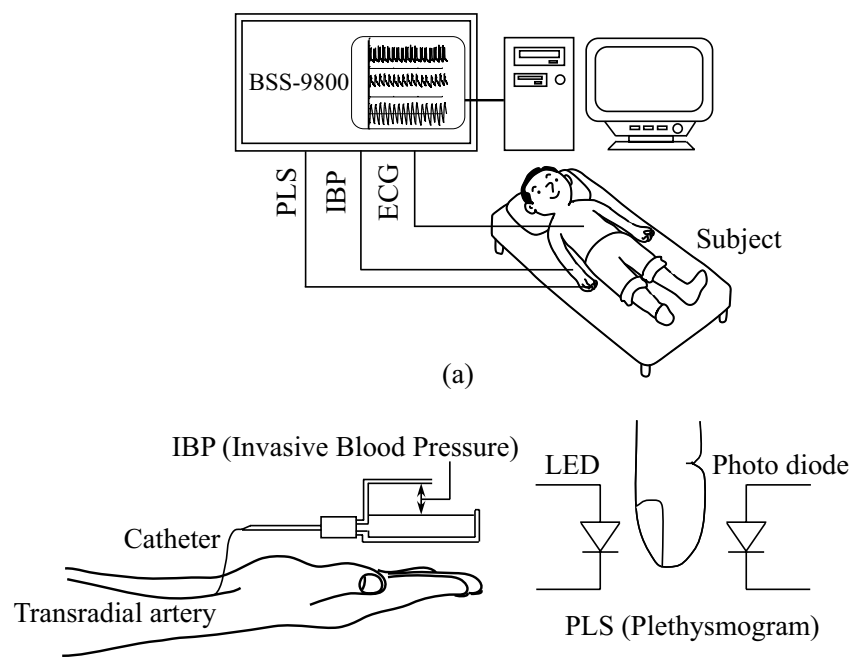

(b)

Fig. 4. (a) Experimental apparatus. (b) IBP is measured at transradial artery of wrist using a catheter, and PLS is measured with the ipsilateral forefinger.

after thirty minutes passed since the patient recovered from the anesthetic state.

Fig. 5 presents an example of the measured data of patient A. This figure plots the electrocardiogram, the arterial pressure, and the plethysmogram. Because the data were affected by some artifacts such as light and mechanical stimulation on the patient's hand, the digital filter was used to regulate frequency characteristics of arterial pressure and the plethysmogram. The arterial pressure is filtered out through a second-order IIR low-pass filter $(6[\mathrm{~Hz}])$ and a first-order IIR high-pass filter $(0.3[\mathrm{~Hz}])$, the plethysmogram through an eighth-order FIR low-pass filter $(15[\mathrm{~Hz}])$ and a first-order IIR high-pass filter $(0.3[\mathrm{~Hz}])$. The arterial pressure and the plethysmogram were divided into some periods based on the R-wave of ECG, and the time $t_{0}$ is defined as the beginning of the period. $d P_{b}(t), d P_{l}(t), d \dot{P}_{l}(t), d \ddot{P}_{l}(t)$ of (12) were then calculated. As the data are extracted beat-to-beat, temporal changes in impedance over time should be observable.

\section{B. Impedance Estimation Results}

We analyzed the response of the vascular smooth muscle induced by changes of transmural pressure [18] to determine the change of the arterial wall impedance due to arm position. Here, the arm position changes in a vertical direction, which results in changes of the relative position between the finger and the heart.

The myogenic response, which is a peripheral mechanism dependent on arm position change, has been investigated [19], [20]. Bayliss reported that the tension in the vascular smooth muscle was caused by a rise in the transmural pressure, and the relaxation of the vascular smooth muscle was caused by a fall in the transmural pressure [21]. It was previously confirmed that stiffness increased (compliance decreased) when arterial pressure rose, while stiffness decreased (compliance
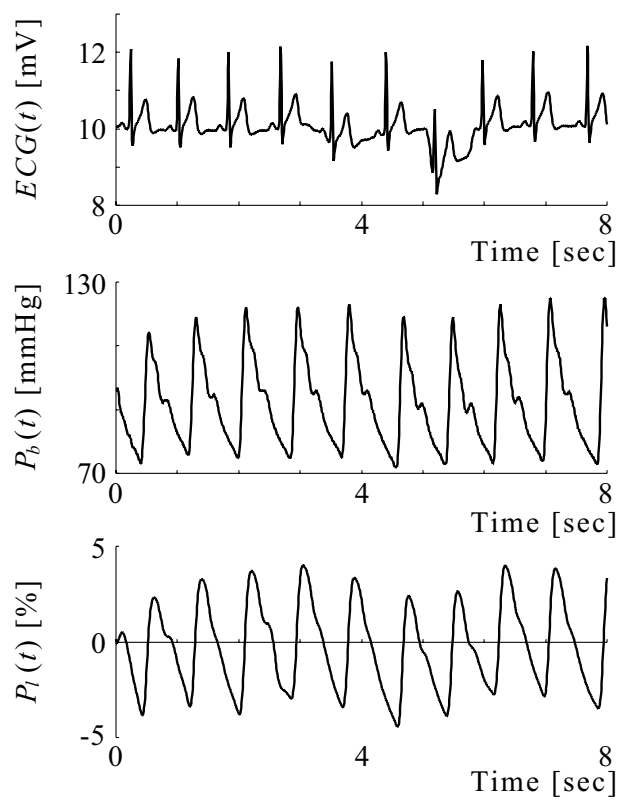

Fig. 5. Examples of the measured signals

increased) when arterial pressure fell. In this paper, we estimated impedance parameters according to the arm position and compared the estimated stiffness with one by the conventional research to ascertain the accuracy of the proposed model.

Fig. 6 shows an example of plethysmogram and arterial pressure used for impedance estimation of Patient A, where the figures (a), (b), (c) and (d) represent the time histories of plethysmogram, $d P_{l}(t)$, velocity of the plethysmogram, $d \dot{P}_{l}(t)$, an accelerated plethysmogram, $d \ddot{P}_{l}(t)$, and arterial pressure, $d P_{b}(t)$, respectively. The solid and dotted lines indicate the measured pressure and the estimated pressure obtained by (12). We found from Fig. 6 that vascular characteristics can be expressed with the proposed model in (12).

Impedance parameters estimated with contribution ratio over 0.9 are plotted in Fig. 7, where the shaded areas corresponds to the states in which the arm was raised. The contribution ratio indicates whether the model can adequately describe vascular characteristics. Significant changes were observed in the estimated impedance parameters. The stiffness decreases when the arm is raised, inversely, the stiffness increases when arm is lowered. Such events have been mentioned in the conventional research [19], [20], [21]. Although the contribution ratio becomes low because the data included noise when the arm position was changed, the usefulness of the proposed model is validated.

Fig. 8 presents an example of Lissajou's figure. The inclination of the figure gives an approximation of blood vessel compliance. It can be seen also that the data estimated from impedance parameters agree well with the measured data. 


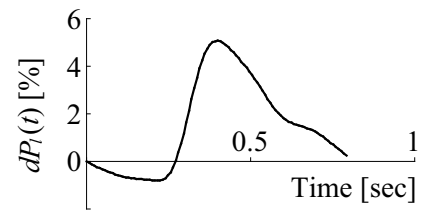

(a)

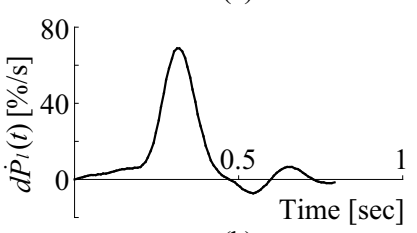

(b)

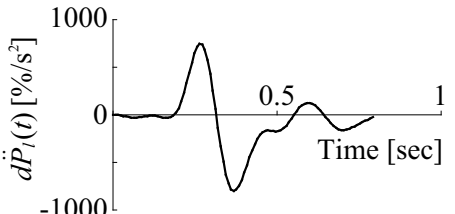

(c)
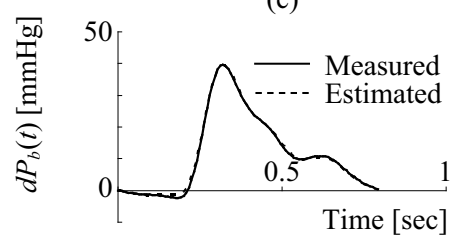

(d)

Fig. 6. Example of the plethysmogram and the invasive blood pressure used for the impedance estimation

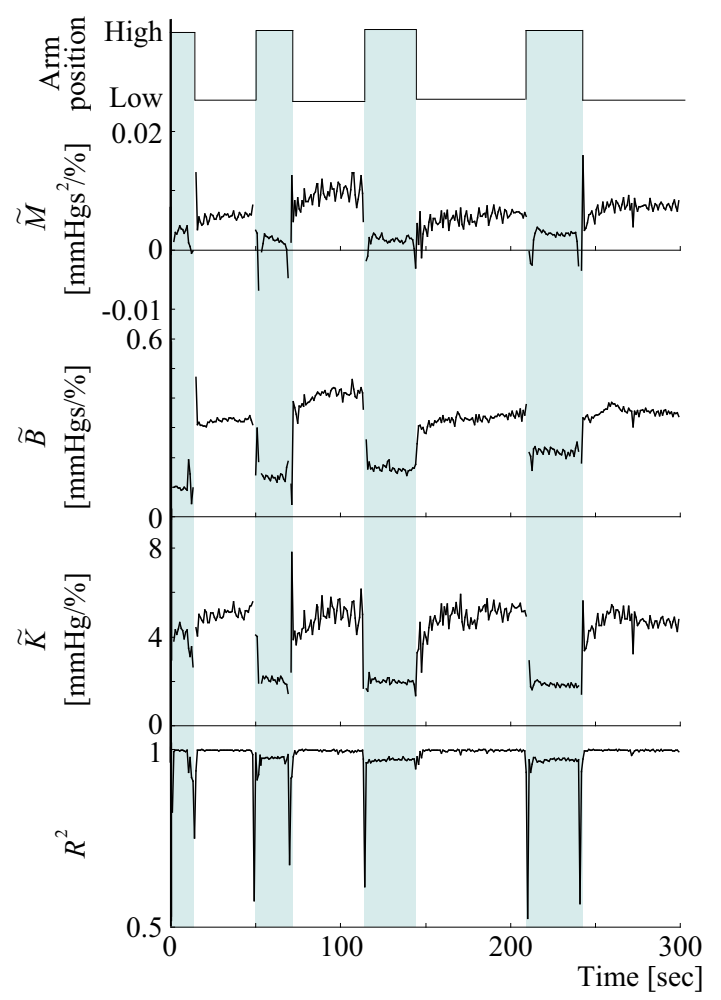

Fig. 7. Estimated impedance parameters

\section{IMPEDANCE MONITORING OF ARTERIAL WALL}

The conditions of a patient must be monitored during surgical operations to appropriately treat the patient with precise timing. The proposed method has the potential to applying to a monitoring system for such vascular conditions. We therefore attempted to monitor changes of arterial wall impedance.

The proposed method is applied to monitoring the impedance of the arterial wall in the following two kinds of surgical operations as follows.

1) Endoscopic transthoracic sympathectomy for hyperhidrosis patients (Patient A) If a blood vessel contracts

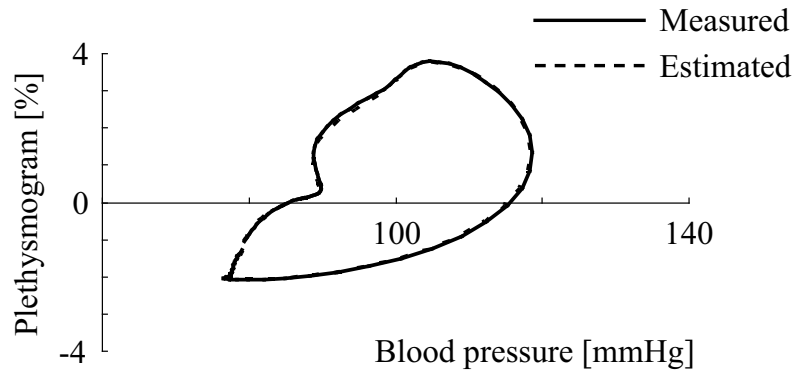

Fig. 8. Lissajou's figure using the proposed model

due to stimulation from sympathetic nerves, the palms and armpits will perspire. In this operation, the sympathetic nerve on the sides of the backbone is interrupted using a clip to stop the perspiration, and a blood vessel become compliant. After that, strong stimulations are not given in a general way.

2) Partial resection of the tongue cancer (Patient B) Various stimulations were given during operations, such as electric knife.

Fig. 9 (a) presents the change of estimated impedance properties during endoscopic transthoracic sympathectomy for a contribution ratio exceeding 0.9 . The sympathetic nerves of the subject were interrupted by clip at the shaded areas. The blood vessels gradually became stiff because a doctor stimulated the patient's tissues to find the sympathetic nerves. After the sympathetic nerves were interrupted, the blood vessels became compliant. During the latter part of the operation, the subject felt pain because the effect of anesthesia started to wear off. The estimated vascular conditions corresponded well with the operational events. The contribution ratio was sometimes reduced because the measuring apparatus failed to measure the data. The accuracy of impedance estimates was evaluated by the contribution ratio.

Fig. 9 (b) depicts the result of a partial resection of the tongue cancer. The patient had a laryngoscope inserted at 200 [sec], and a tracheal tube at 500[sec]. Strong stimulations were given twice for 2000 to 3000 [sec], and the effect of the anesthesia wore off after $5000[\mathrm{sec}]$. The changes of arterial wall impedance corresponded with events in each subject.

\section{CONCLUSION}

This paper proposed a novel technique using mechanical impedance for modeling the dynamic characteristics of the arterial wall, and a method for estimating vascular conditions beat-to-beat. The impedance parameters were estimated by fundamental experiments during vertical movement of the arm, and it has been shown that the changes of impedance parameters correspond to the changes of the arm position. The proposed method was then applied to the monitoring of arterial wall impedance during surgical operations. The experiments revealed that the changes of impedance corresponded with events during operations. 


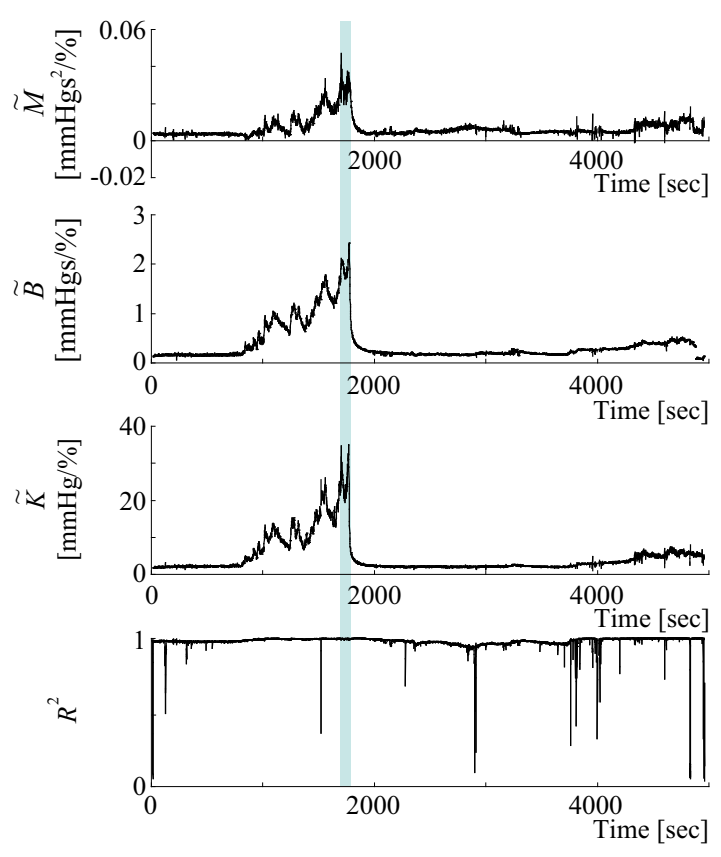

(a) Patient A
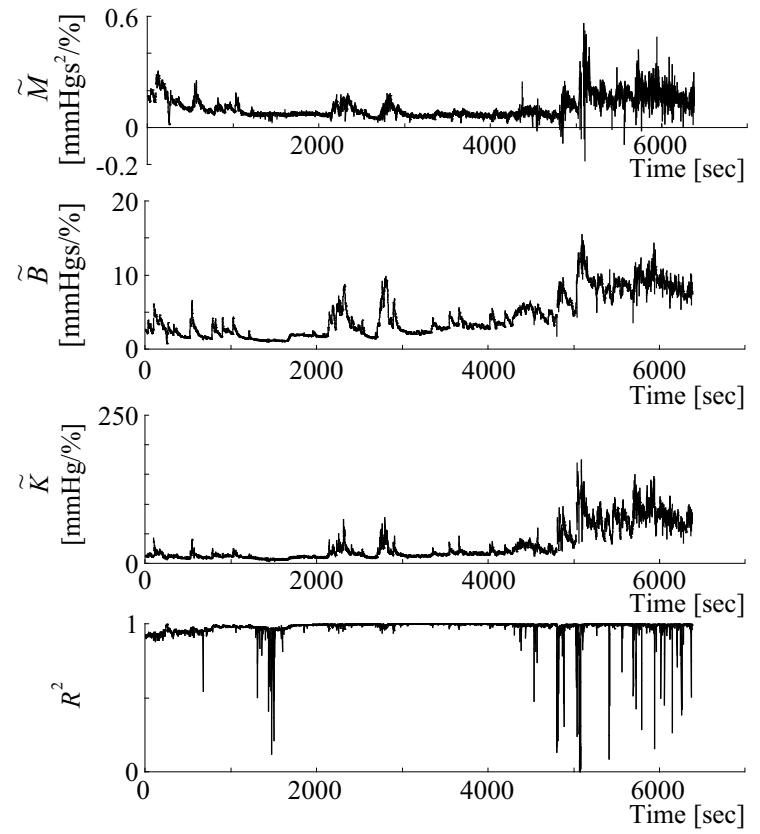

(b) Patient B

Fig. 9. Impedance monitoring of arterial wall during operations

In the experiments, this paper used the invasive blood pressure to estimate the arterial wall impedance. Future research will be directed to use a non-invasive blood pressure for establishing a non-invasive technique. We will then attempt to evaluate and discriminate various vascular conditions by using estimated impedance parameters and biological signals.

\section{ACKNOWLEDGMENT}

The authors would like to thank T.Ukawa and K.Tone of the NIHON KOHDEN Co., Ltd for making available clinical data and for their precious suggestions.

This work was supported by Grant-in-Aid for Scientific Research of Japan Society for the Promotion of Science (15008279).

\section{REFERENCES}

[1] W.W.Nichols, et al.: McDonald's Blood Flow in Arteries: Theoretical, Experimental and Clinical Principles, 4th ed, Arnold, London, 1998.

[2] F.A.Mussa-Ivaldi, N.Hogan, and E.Bizzi: Neural, mechanical and geometrical factors sub-serving arm posture in humans, Journal of Neuroscience, Vol.5, No.10, 2732-2743, 1985.

[3] J.M.Dolan, M.B.Friendman, and M.L.Nagarka: Dynamics and loaded impedance components in the maintenance of human arm posture, IEEE Transaction on Systems, Man and Cybernetics, 23, 3, 698-709, 1993.

[4] T.Tsuji, P.G.Marossa, K.Goto, and K.Ito: Human hand impedance characteristics during maintained posture, Biological Cybernetics, 72, 457-485, 1995.

[5] T.Tsuji and M.Kaneko: Estimation and Modeling of Human Hand Impedance during Isometric Muscle Contraction, Proceedings of the ASME Dynamic Systems and Control Division, DSC-Vol.58, 575-582, Atlanta, 1996.

[6] H.Oka, T.Irie, K.Yasuhara, and T.Yamamoto: Transducer for Measuring Skin Stiffness, Sensors and Materials, Vol.4, No.6, 337-349, 1993.

[7] J.C.Greenfield, et al.: Relation between pressure and diameter in the ascending aorta of man, Circ Res, No.10, 778-781, 1962
[8] K.Katayama, M.Shimoda, J.Maeda, and T.Takemiya: Endurance Exercise Training Increases Peripheral Vascular Response in Human Fingers, The Japanese Journal of Physiology, Vol.48, No.5, 365-371, 1998.

[9] Y.Sawada, G.Tanaka, and K.Yamakoshi: Normalized pulse volume(NPV) derived photo-plethysmographically as a more valid measure of the finger vascular tone, International Journal of Psychophysiology, Vol.41, 1-10, 2001.

[10] N.Saeki, M.Kawamoto, and O.Yuge: Quantitive view of peripheral circulation, 30th International Educational and Scientific Symposium, Society of Critical Care Medicine, Moscone Center, San Francisoco, CA, USA February 10-14, 2001.

[11] A.Mascaro and H.Asada: Photoplethysmograph Fingernail Sensors for Measuring Finger Forces Without Haptic Obstruction, IEEE Transactions on robotics and automation, Vol.17, No.5, 698-708, 2001.

[12] R.Kato, J.Sato, T.Iuchi, and Y.Higuchi: Quantitative determination of arterial wall mechanics with pulse oximetric finger plethysmography, Journal of Anesthesia, Vol.13, 197-204, 1999.

[13] Y.Murahara, K.Sakamoto, H.Kanai, and T.Arai: Estimation of the Arterial Stenosis by Means of Blood Pressure Waveform, Medical Physics, Vol.27, No.6, p.1421, Chicago, 2000.

[14] W.Kahle, H.Leonhardt, and W.Platzer: Taschenatlas der Anatomie, Georg Thieme Verlag, 1986.

[15] C.Stefanadis, C.Vlachopoulos, P.Karayannacos, et al.: Effect of vasa vasorum flow on structure and function of the aorta in experimental animals, Circulation, 91, 2669-2687, 1995.

[16] R.A.Day and A.L.Underwood: QUANTITATIVE ANALYSIS 4th Edition, Prentice-Hall, 1980.

[17] T.Aoyagi and T.Ukawa: Theorem and structure of Pulseoximeter, Clinical Engineering, Vol.7, No.2, 102-110, 1996.

[18] S.Mellander: Myogenic mechanisms in local vascular control, Acta Physiol Scand Suppl, 25-42, 1988.

[19] J.Maeda, T.Takemiya, M.Shimoda, J.Suzuki, and H.Komiya: Local vascular response induced by arm position change: The related myogenic mechanism and veno-arteriolar reflex in humans, Advances in Exercise and Sports Physiology, No.3, 67-73, 1997.

[20] T.Takemiya, J.Maeda, J.Suzuki, Y.Nishihira, and M.Shimoda: Differential digital photoplethysmographic observations of finger vascular exponential response to the arm position changes in humans, Advances in Exercise and Sports Physiology, No.2, 83-90, 1996.

[21] W.M.Bayliss: On the local reactions of the arterial wall to changes of internal pressure, J Physiol, No.28, 220-231, 1902. 